\title{
Audiogenic seizure tests of ethanol addiction/withdrawal are unaffected by previous tests
}

\author{
MILTON A. TRAPOLD \\ Memphis State University, Memphis, Tennessee 38152
}

\begin{abstract}
In previous work (Trapold \& Sullivan, 1979), we reported the results of a new method of deriving a quantitative index of the severity of the withdrawal process in ethanol-addicted rats. Initial results suggest that the audiogenic seizure response that provides this index is not seriously affected by the subjects' past history of testing for this response. The paper presents the results of two further experiments, one involving alcohol-naive rats, the other involving alcohol-addicted rats; these results further support the conclusion that subjects may be repeatedly tested for audiogenic seizure without fear that the seizure elicited on a particular test will be seriously influenced by the subjects' past history of such testing.
\end{abstract}

In a recent paper (Trapold \& Sullivan, 1979), we reported initial results of a technique for rapidly assessing the severity of withdrawal from ethanol in rats. The technique involves subjecting the rat to the sound of jingling keys for a period of time and scoring the strength of the seizure activity thereby elicited on a scale from 0 (no seizure activity) to 4 (seizure activity resulting in death). Following an extended period of continuous alcoholization, both the percentage of subjects showing seizure activity and the mean strength of elicited seizure activity showed a smooth increase from nearly 0 at the beginning of withdrawal through a maximum between 6 and $12 \mathrm{~h}$ postwithdrawal, and then a smooth decrease back toward 0 again. The maximum frequency and strength of seizures were higher when subjects were withdrawn following a long duration of continuous alcoholization than following a short duration. Furthermore, the administration of diazepam temporarily suppressed seizures.

For maximum usefulness in studying variables that may affect the severity, the time course, or other properties of withdrawal, one would like to be able to use a withdrawal-assessing probe stimulus like this repeatedly in the same subject without fear that the reaction elicited on one particular application of the probe may depend on carryover effects from previous applications of the probe (e.g., habituation or sensitization). In the previous report, we tentatively concluded that keyjingling seizures do not show any important degree of such carryover. Repeated testing across several cycles of alcoholization and withdrawal in the same subjects yielded seizure-based withdrawal functions that did not depend upon the amount of prior experience the subjects had had with the probe stimulus, but which did vary systematically with the duration of alcoholization preceding a particular withdrawal.

The present paper reports two additional experi- ments that were conducted to further determine whether audiogenic seizure measures of ethanol withdrawal are seriously influenced by the organism's prior history of seizure testing. A secondary purpose of the second experiment was to determine if the functions relating seizure percentage and strength to time since withdrawal would be similar following a method of alcoholization different from that employed in the earlier experiment. The earlier experiment employed a modified polydipsia procedure to induce subjects to consume large quantities of alcohol, whereas Experiment 2 here employed a liquid diet technique.

\section{EXPERIMENT 1}

Experiment 1 was designed to assess whether audiogenic seizure frequency and strength in alcohol-naive subjects change in any systematic way when subjects are tested repeatedly once per day.

\section{Method}

Subjects. The subjects were 30 male albino rats approximately 7 months old. Each subject was housed individually in a standard $18 \times 18 \times 25 \mathrm{~cm}$ high home cage and was removed only for seizure testing. Food and water were freely available in the home cage for the first 8 days of the experiment. For the next 4 days, only water was available.

Apparatus. Seizure tests were conducted in a $45 \times 25 \times 15 \mathrm{~cm}$ high opaque plastic chamber with a wire-mesh top. The seizureeliciting sound was produced by a ring of 12 brass keys suspended 12 or $48 \mathrm{~cm}$ (see below) above the top of the center of the test chamber on the end of a metal rod that was driven up and down by an electric motor at the rate of 1,715 complete vertical displacement cycles per minute through a distance of $1.25 \mathrm{~cm}$

Procedure. Subjects were randomly divided into two groups of 15 each. Group R (repeated testing) was then subjected to a single seizure test each day for 11 consecutive days at the $12-\mathrm{cm}$ key-to-chamber distance, and Group I (intermittent testing) was subjected to such a seizure test only on Days 1,9,10, and 11 . 
The procedure for a seizure test was to remove the subject from its home cage to the portable test chamber, carry the subject and chamber into the test room, place the chamber below the key ring, and then energize the key shaker for 1 min or until a seizure of Strength 2 or greater (see below) occurred.

The activity elicited by the shaking keys typically follows a regular sequence that provides the basis for the scoring scheme. The mildest reaction is simply a noticeable increase in normal locomotor activity. This sometimes gives way to "rapid running," a series of frantic staccato bursts of locomotor activity and scrabbling at the chamber top. Rapid running typically persists for some seconds. Sometimes the rapid running simply ceases, but on other occasions it gives way to "thrashing," in which the subject undergoes prolonged wild running punctuated by a series of severe phasic whole-body muscular contractions that have the effect of propelling the subject more or less randomly about the chamber as its limbs strike the floor or walls. In our procedure, as soon as the subject enters the thrashing stage, the key-jingling stimulus is terminated. Sometimes thrashing ceases shortly after the termination of the stimulus, but on other occasions it continues for some seconds and then gives way to a "contraction" phase, in which the subject lies relatively motionless on its side or back and undergoes a prolonged spastic whole-body muscular contraction that has the typical result of pulling the head and forelimbs forward against the ventral surface and of either pulling the hindlimbs tight against the ventral surface or of extending them rigidly tailward. The contraction phase, which lasts from a few seconds to $1 \mathrm{~min}$, is then typically followed by a period of smaller localized spasms (e.g., hindlegs, front legs, jaw) and then by a period of flaccid immobility. In very severe seizures, the rapid-running and thrashing phases are of ten so brief as to be essentially unobservable, the subject going directly into the contraction phase.

The rules for scoring seizure activity were as follows: $0=$ no seizure; 1 = rapid running; 2 = thrashing; 3 = contraction; $4=$ death. In this experiment, no subject died as a direct result of a test-induced seizure, so we never had an occasion to employ a score of 4 . This scoring scheme is easily learned by observers, and periodic reliability checks in our laboratory have consistently yielded better than $95 \%$ agreement between two independent observers, with all disagreements of the 1-2 or 2-3 type.

\section{Results and Discussion}

In the case of both the percentage of subjects yielding any seizure activity (score of 1 or greater) and the mean strength of elicited seizure (including 0s), Group R did not differ significantly from Group I, and neither group showed any systematic change in either measure across Test Days 1-11. For Group R, percentage seizures on the common test days $1,9,10$, and 11 were .47 , $.47, .47$, and .53 , respectively; for Group I, these scores were $.40, .40, .40$, and .47 . For Group $\mathrm{R}$, mean seizure strength on Days $1,9,10$, and 11 were $.93,1.26, .93$, and 1.40; for Group I, these scores were .93, 1.00, .80 , and 1.00 .

The results of this experiment suggest that neither the likelihood of the key jingling eliciting a seizure nor the strength of the seizure elicited is strongly biased one way or the other by the repeated testing at the rate of 1 test/day. That conclusion is consistent with the conclusion reached in the earlier report based upon multiplewithdrawal seizure test series in the same subjects.

\section{EXPERIMENT 2}

The primary purpose of Experiment 2 was to determine more systematically whether the results obtained with the audiogenic seizure measures of ethanol withdrawal depend upon the frequency with which the subject has been exposed to the key-jingling test procedure in the past.

\section{Method}

Subjects. Subjects were 33 male Sprague-Dawley albino rats, approximately 6 months old. Eight subjects died during the course of the experiment. The method for handling the data on the lost subjects is described below.

Apparatus and Procedure. The apparatus and procedure for conducting seizure tests were identical to those described in Experiment 1.

All subjects were first reduced to $80 \%$ of their freefeeding body weights via limited access to Purina Lab Chow. They then were randomly divided into three groups and subjected to a series of treatments and tests, as summarized in Table 1.

Each group was run through a series of four successive cycles of alcoholization and withdrawal. The first alcoholization was 1 day long; the second, third, and fourth were each 5 days long. Each alcoholization was followed immediately by an alcohol-withdrawal period. The first three withdrawals were $48 \mathrm{~h}$ long; the fourth was $72 \mathrm{~h}$ long. Group 1 was subjected to a series of seizure tests during each of the four withdrawals at the times indicated in Table 1. Group 2 received the seizure test series during only the third and fourth withdrawals, and Group 3 only during the fourth withdrawal. Thus, the seizure tests during the third withdrawal allowed comparison

Table 1

Sequence of treatment Phases and Number as Subjects for Each of the Three Groups of Experiment 2

\begin{tabular}{|c|c|c|c|c|c|c|c|}
\hline \multirow[b]{2}{*}{ Successive Treatment Phases } & \multirow{2}{*}{$\begin{array}{l}\text { Alcoholization/Withdrawal } \\
\text { Duration Test Points }\end{array}$} & \multicolumn{2}{|c|}{ Group 1} & \multicolumn{2}{|c|}{ Group 2} & \multicolumn{2}{|c|}{ Group 3} \\
\hline & & $\mathbf{n}$ & $I$ & $\mathrm{n}$ & I & $\mathrm{n}$ & I \\
\hline Alcoholization/Withdrawal 1 & 1 day $/ 2$ days & 11 & $\mathbf{Y}$ & 11 & $\mathbf{Y}$ & 11 & $\mathrm{Y}$ \\
\hline Withdrawal Test 1 & $0,3,6,12,24,48 \mathrm{~h}$ & 11 & $\mathbf{Y}$ & 11 & $\mathbf{N}$ & 11 & $\mathrm{~N}$ \\
\hline Alcoholization/Withdrawal 2 & 5 days $/ 2$ days & 11 & $\mathrm{Y}$ & 11 & $\mathrm{Y}$ & 11 & $\mathrm{Y}$ \\
\hline Withdrawal Test 2 & $0,3,6,9,12,15,18,21,24,48 \mathrm{~h}$ & 11 & $\mathrm{Y}$ & 11 & $\mathrm{~N}$ & 11 & $\mathrm{~N}$ \\
\hline Alcoholization/Withdrawal 3 & 5 days $/ 2$ days & 11 & $\mathbf{Y}$ & 11 & $\mathrm{Y}$ & 11 & $\mathrm{Y}$ \\
\hline Withdrawal Test 3 & $0,3,6,9,12,18,21,24,48 \mathrm{~h}$ & 11 & $\mathrm{Y}$ & 11 & $\mathrm{Y}$ & 11 & $\mathrm{~N}$ \\
\hline Alcoholization/Withdrawal 4 & 5 days/3 days & 10 & $\mathrm{Y}$ & 8 & $\mathrm{Y}$ & 11 & $\mathrm{Y}$ \\
\hline Withdrawal Test 4 & $0,3,6,12,24,48,72 \mathrm{~h}$ & 9 & $\mathrm{Y}$ & 7 & $\mathrm{Y}$ & 9 & $\mathrm{Y}$ \\
\hline
\end{tabular}

Note-Y means a group was included in the particular phase indicated on the left; $N$ means a group was not included. All three groups received all four alcoholizations and withdrawals. They differed only in whether they received a series of seizure tests during a withdrawal or not. 
of subjects with identical alcoholization histories, but with one group being tested for the first time (Group 2) and another already having been through two prior series of seizure tests totaling 12 tests (Group 1). The seizure tests during the fourth withdrawal provided a similar comparison between subjects being tested for the first time (Group 3) and subjects that had one prior test series (Group 2) or subjects that had three prior test series (Group 1).

All alcoholization in this experiment was conducted by giving subjects free access to a single fluid that constituted the only source of nourishment and fluid and that contained a known quantity of alcohol. This liquid diet was composed of $100 \mathrm{~g}$ of Purina Nurse Gro milk replacer, $50 \mathrm{~g}$ of sucrose, $100 \mathrm{ml}$ of $95 \%$ ethanol, $1 \mathrm{ml}$ of Polyvisol multiple-vitamin supplement, and tap water to a total of 1 liter.

During withdrawal periods, subjects had free access to water in the home cage plus a maintenance diet of $12 \mathrm{~g}$ of Purina Lab Chow per day.

\section{Results and Discussion}

Table 2 summarizes the mean body weight and mean daily alcohol consumption for each of the four alcoholization phases of this experiment.

The changing number of subjects reflects the animals that died during the experiment. The rule used throughout was to include the subject's data in only those phases of the experiment that it completed, as listed in Table 1.

Table 2 shows that the three groups had quite comparable body weights and levels of alcohol consumption throughout the four alcoholizations. The daily ethanolconsumption levels of about $14-18 \mathrm{~g} / \mathrm{kg} / \mathrm{day}$ were in the range previously reported to produce physical dependence in the rat (Hunter, Riley, Walker, \& Freund, 1975; Majchrowicz \& Hunt, 1976; Mello, 1973).

Figure 1 presents results of seizure testing during Withdrawals 1 and 3, and Figure 2 presents the results from Withdrawal 4 . The curve produced by Group 1 on Withdrawal 3 was eventually identical to that from Withdrawal 4, and so it is not presented. In both Figure 1 and Figure 2, the upper panel plots the percentage of subjects showing any seizure activity, and the lower panel plots the mean strength of seizure elicited.

In general, the function relating elicited seizures to time in alcohol withdrawal was very similar to that reported previously with this technique (Trapold \& Sullivan, 1979) and to that reported by Goldstein (1977) for tail-lift elicited seizures in Swiss-Webster mice. Seizures were at 0 strength immediately upon

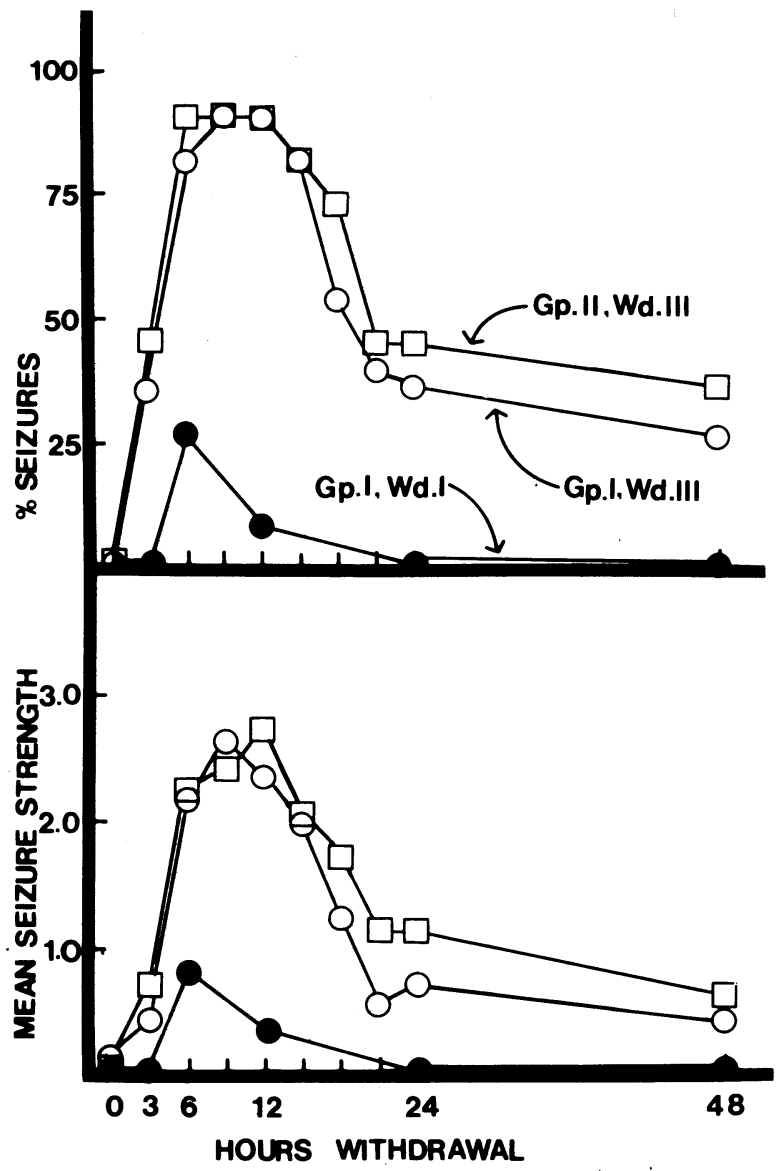

Figure 1. Seizure percentage and mean seizure strength as a function of time in withdrawal for Groups 1 and 2 during Withdrawals 1 and 3.

entry into withdrawal, then quickly rose to a maximum at $6-12 \mathrm{~h}$, and then declined toward 0 again over the next several days.

The most important observation in these results for the present purposes is that the form of the seizurewithdrawal function was essentially unaffected by whether subjects had been through one, two, or three prior seizure test series. The differences among the curves obtained in Withdrawal 3 and in Withdrawal 4 were very slight, implying that the elicited seizure response is not seriously influenced by the subject's prior history of seizure testing.

Table 2

Mean Body Weight and Mean Alcohol Consumption During the Four Alcoholizations for Each of the Three Groups of Experiment 2

\begin{tabular}{|c|c|c|c|c|c|c|c|c|c|}
\hline \multirow[b]{2}{*}{ Alcoholization } & \multicolumn{3}{|c|}{ Group 1} & \multicolumn{3}{|c|}{ Group 2} & \multicolumn{3}{|c|}{ Group 3} \\
\hline & $\mathrm{n}$ & W & E & $\mathrm{n}$ & W & $\mathrm{E}$ & $\mathrm{n}$ & W & $\mathbf{E}$ \\
\hline $\begin{array}{l}1 \\
2 \\
3 \\
4 \\
\end{array}$ & $\begin{array}{l}11 \\
11 \\
11 \\
10 \\
\end{array}$ & $\begin{array}{l}306 \\
323 \\
312 \\
312 \\
\end{array}$ & $\begin{array}{l}14.9 \\
18.1 \\
16.4 \\
17.1 \\
\end{array}$ & $\begin{array}{r}11 \\
11 \\
11 \\
8 \\
\end{array}$ & $\begin{array}{l}316 \\
331 \\
310 \\
310 \\
\end{array}$ & $\begin{array}{l}16.7 \\
16.4 \\
15.9 \\
16.8 \\
\end{array}$ & $\begin{array}{l}11 \\
11 \\
11 \\
11 \\
\end{array}$ & $\begin{array}{l}311 \\
325 \\
310 \\
318\end{array}$ & $\begin{array}{l}14.2 \\
18.2 \\
16.6 \\
17.5\end{array}$ \\
\hline
\end{tabular}

Note $-W=$ mean weight in grams; $E=$ mean grams ethanol consumed per kilogram body weight per day. 


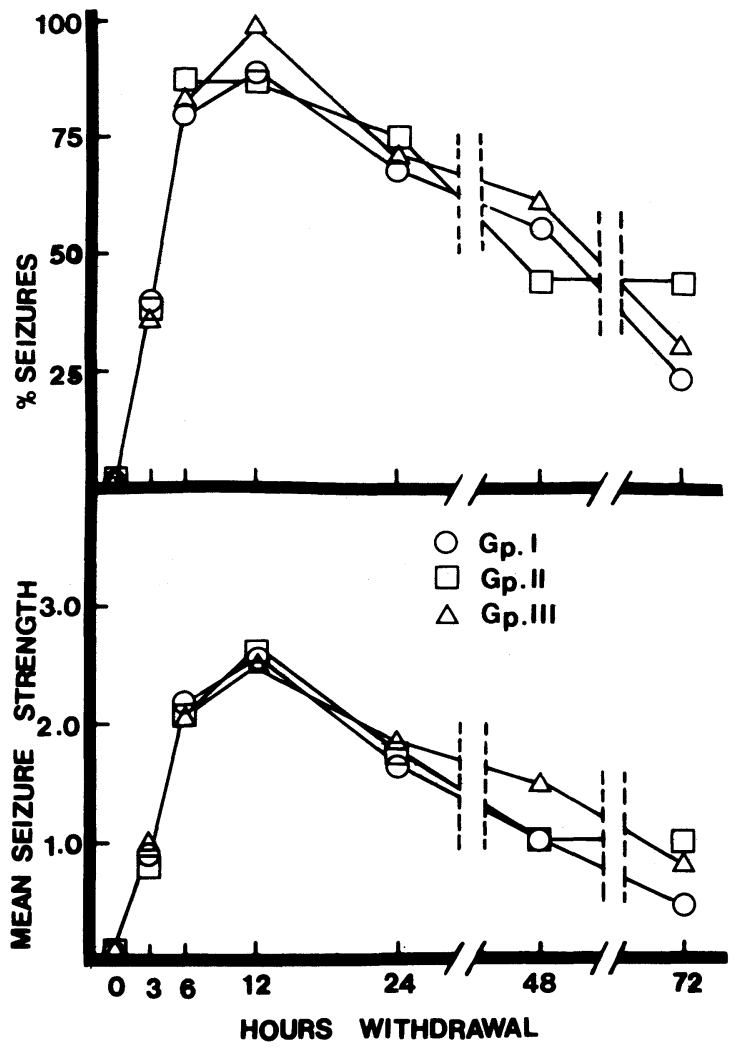

Figure 2. Seizure percentage and mean seizure strength as a function of time in withdrawal for Groups 1, 2, and 3 during Withdrawal 4.

Another noteworthy fact from Figure 1 is that only 1 single day of exposure to alcohol was sufficient to produce a slight and short-lived elevation of seizure strength during subsequent withdrawal. A similar result has been reported previously (Goldstein, 1972).

\section{GENERAL DISCUSSION}

Overall, the results of these experiments add further weight to the conclusion drawn tentatively by Trapold and Sullivan (1979) that the elicited seizure technique for assessing alcohol withdrawal is not seriously affected by prior seizure tests. Seizure tests can evidently be used repeatedly on the same subject without one's having to be concerned that earlier tests may importantly influence or complicate interpretation of subsequent tests.

\section{REFERENCES}

Goldstein, D. B. Characteristics of ethanol physical dependence. In T. Thompson \& K. R. Unna (Eds.), Predicting dependence liability of stimulant and depressant drugs. Baltimore: University Park Press, 1977.

Hunter, B. E., Riley, J. N., Walker, D. W., \& Freund, G. Ethanol dependence in the rat: A parametric analysis. Pharmacology, Biochemistry and Behavior, 1975, 3, 619-629.

Majchrowicz, E., \& Hunt, W. A. Temporal relationship of the induction of tolerance and physical dependence after continuous intoxication and maximum tolerable doses of ethanol in rats. Psychopharmacologion, 1976, 50, 107-112.

Mello, N. A review of methods to reduce elcohol addiction in animals. Pharmacology, Biochemistry and Behavior, 1973, 1, 89-101.

Trapold, M. A., \& Sullivan, H. L. Assessment of the ethanol withdrawal state in the rat by means of repeatedly elicited audiogenic seizures. Bulletin of the Psychonomic Society, 1979, 14, 301-303.

(Received for publication June 5, 1980.) 